\title{
Outcome of patients with ST segment elevation myocardial infarction (STEMI) following percutaneous transluminal coronary angioplasty: a retrospective study
}

\author{
V Abeysuriya ${ }^{1}$, L G Chandrasena ${ }^{2}$, A Kasturiratne ${ }^{1}$, V S Hettiarachchi ${ }^{2}$, A R Wickremasinghe ${ }^{1}$ \\ (Index words: survival, STEMI, PTCA)
}

\begin{abstract}
Objectives To study the characteristics and long term outcome of patients who had segment elevation myocardial infarction (STEMI) and treated with PTCA in a fee levying hospital in Sri lanka.

Methods A retrospective study was conducted among patients diagnosed with STEMI and treated with PTCA in a fee levying private hospital in Colombo from 1st January 2009 to 1st November 2012. Details of patients were obtained from medical records and the survival status, cause of death and date of death where relevant, were obtained from records, patients or close relatives.

Results 197 patients (153 men; 77.7\%) were included. More than $50 \%$ had a history of diabetes, dyslipidaemia or hypertension. The three year survival was $82.7 \%(95 \% \mathrm{Cl}$ : 77.9\%-90.5\%). Based on the Cox's Proportional Hazards model, site of arterial occlusion (proximal vs distal segment of left anterior descending artery [LAD] was significantly associated with mortality due to all causes [HR 10.98; $95 \% \mathrm{Cl}: 1.09-110.20]$. Low ejection fraction, not on regular medication and delay of more than 3 hours between onset to door time were associated with death due to cardiovascular causes in patients whose right coronary artery or left circumflex artery was the culprit artery.

Conclusions The three year survival of patients with STEMI and who had PTCA was $83 \%$. Patients with proximal LAD occlusion were 11 times more likely to die within 3 years of PTCA as compared to those who had a distal LAD occlusion.
\end{abstract}

Ceylon Medical Journal 2014; 59: 118-123

\section{Introduction}

Cardiovascular diseases remain a major cause of death worldwide. Over the last two decades, cardiovascular mortality rates have declined in many high-income countries. During the same time, cardiovascular deaths and disease incidence have increased at an alarmingly high rate in low-and middle-income countries [1].

Acute coronary syndrome encompasses a spectrum of coronary artery diseases, including unstable angina, ST-elevation myocardial infarction (STEMI) and non ST-elevation myocardial infarction [2]. The most severe form of acute coronary syndrome (ACS) is STEMI [3].

The demographics in Sri Lanka are changing to an older and ageing population. The country has to deal with escalating health care costs as a result of increasing rates of non-communicable diseases (NCDs). NCDs accounts for $85 \%$ of ill health, disability and early deaths in Sri Lanka [4].

Primary preventive strategies play a major role in reducing STEMI. But due to the complex risk model involving modifiable and non-modifiable risk factors and their interactions, reducing the burden of noncommunicable diseases is more difficult. Therefore evidence based secondary prevention in the short and medium term is important. The objective of this study was to assess the outcome of patients with STEMI after Percutaneous Transluminal Angioplasty (PTCA) treatment at a fee levying hospital in Colombo.

\section{Methods}

This retrospective study was carried out at a fee levying hospital in Colombo. The study population comprised of patients diagnosed with STEMI treated with PTCA in the hospital from $1^{\text {st }}$ January 2009 to $30^{\text {th }}$ October 2012. Patients were selected from the Emergency Treatment Unit (ETU) admission register at the hospital.

Inclusion criteria for selection of patients were having clinical symptoms of angina, ST segment elevation more than $1 \mathrm{~mm}(>0.1 \mathrm{mV})$ in two or more consecutive leads in traditional 12 lead ECG, and positive bio markers of myocardial damage (hFABP and/or Trop I and/or Trop $\mathrm{T})$. The diagnosis was confirmed on positive coronary angiogram findings by the on call consultant cardiologist.

${ }^{1}$ Department of Public Health, Faculty of Medicine, University of Kelaniya and ${ }^{2}$ Department of Cardiology, Nawaloka Hospitals PLC, Colombo 2, Sri Lanka.

Correspondence: VA, e-mail: <visulasrilanka@hotmail.com>. Received 2 July 2014 and revised version accepted 13 September 2014. Competing interests: none declared. 
Exclusion criteria were patients who had unstable angina or LBBB (associated with ST elevation) and other cardiac problems such as cardiomyopathy and valvular heart diseases, patients who experienced out-of-hospital cardiac arrest, patients who failed to have a return of spontaneous circulation (ROSC), and patients who had the first ECG after cardiopulmonary resuscitation.

During the study period there were 207 STEMI patients admitted to Emergency Treatment Unit (ETU) of the hospital. Out of them, 10 patients had been transferred to a government hospital for further treatment due to financial difficulties and were excluded from the analysis. Records of 197 patients were analysed.

Data were obtained from case records, discharge diagnosis card summaries and hospital databases. Details not mentioned in available records were obtained from telephone interviews with patients or next of kin or during routine clinic visits.

A data collection sheet was developed to record details of demographic and socioeconomic status, past medical and surgical histories, clinical characteristics, results of relevant biochemical investigations and treatment modalities. Dyslipidaemia was classified according to the National Cholesterol Education Programme Expert Panel on Detection, Evaluation, and Treatment of High Blood Cholesterol in Adults Treatment Panel III-USA (NCEP ATP III) criteria [5]. Diabetes was defined as a fasting plasma glucose $\square 7 \mathrm{mmol} / \mathrm{l}$ (or 126 $\mathrm{mg} / \mathrm{dl}$ ) [6]. Hypertension was defined based on the WHO classification for blood pressure measurements [7]. The severity was ranked according to Killip class classification [8]. Details of coronary angiography findings were obtained from the Hospital angiogram laboratory register. The culprit artery and other lesion sites were recorded from the register. Coronary angiographies were carried out using Philips Model-FD-10. The lesion with the highest degree of stenosis was considered as the culprit lesion. The right coronary artery was divided into proximal, mid and distal segments. The segment of the RCA from its ostium to the origin of the first acute marginal branch was considered the proximal segment; from the origin of first acute marginal branch to that of the last acute marginal branch was considered the mid segment; and from this point onwards as the distal segment. The left anterior descending artery (LAD) was divided into proximal, mid and distal segments. LAD from the ostium to the origin of the first septal branch was considered the proximal segment; the end of the proximal segment to the midpoint (half the distance between the end of the proximal segment to the apex of the LAD) as the middle segment, and below the midpoint was considered as the distal segment.

The EASI (referring to E, A, S and I electrodes of the ECG system) method was used to record the height of ST segment elevation $[9,10]$. The size of ST segment elevation was recorded from thePhilips IntelliVueMP20 monitor to which the EASI electrodes were connected. The machine was able to quantify the ST segment elevation in a relevant lead in less than a minute. ST elevation was measured $60 \mathrm{~ms}$ from the right of the J point; a $1 \mathrm{~mm}$ or greater elevation from the isoelectric line was considered as "elevated" $[11,12]$.

Survival time of patients after the event was obtained during routine clinic visits, telephone interviews and records maintained at the hospital. There were no refusal to provide details during the interviews with the patients at the clinic or telephone conversations. No patients had undergone CABG or re-stenting after initial PTCA. Details were collected on present status of the patient (dead or alive) and cause and exact time of death (due to cardiac or other), where relevant. Cause of death was confirmed from the certified death certificate issued by a consultant physician and or consultant cardiologist or a report issued following an inquest by a hospital.

Normally distributed data were summarised as means, standard deviations and confidence intervals. Kaplan Meier survival curves were generated and Cox's regression model was used to adjust for potential confounding factors. In the Cox's regression model the stepwise procedure was used to retain only significant predictors in the model. Data were analysed using SPSS version 13.0 (SPSS 13.0, Chicago, Illinois, USA) and STATA version 12 (12.0, Texas, USA). A p-value $<0.05$ was considered significant. The study was approved by the Ethics Review Committee of the Faculty of Medicine, University of Kelaniya, Sri Lanka. Permission to conduct the study was obtained from the Institute. All data were kept confidential and data were anonymised.

\section{Results}

Table 1 shows the demographic and clinical characteristics of the study population. The mean age of males was 55.3 years and in females 58.8 years (Table 1). More than $80 \%$ of the patients received intervention less than half an hour after admission to the hospital. Of the patients $37 \%$ presented with profuse vomiting, faintish attacks and blackouts, or abdominal pain without any chest pain. According to Killip class classification $70.9 \%$ were in class I (Table 2$)$. The majority of patients $(n=160)$ had left anterior descending (LAD) artery occlusions.

During the study period there were 23 deaths due to all causes among the 197 patients, of which, 13 were cardiac related deaths. Kaplan-Meier survival curve was generated for all deaths (Figure 1). The 3-year survival probability of these patients from deaths due to all causes was $82.7 \%$ (95\% CI 75.8-89.6), and from cardiovascular related deaths was $89.4 \%$ (95\% CI 83.5 - 95.4).

The commonest cause of death was congestive cardiac faliure $(30.4 \%)$, followed by acute myocardial infarction (26.1\%) (Table 3). More than $50 \%$ of cardiac deaths were due to congestive cardiac failure (Table 3 ). 
Most patients (35.4\%) who had LAD artery lesion died due congestive cardiac failure whereas patients with lesion sites in RCA and left circumflex artery died due acute myocardial infraction and other causes.

Age of the patient, ever having smoked, history of dyslipidaemia, a positive heart fatty acid binding protein (hBFABP), having an occlusion in the proximal segment of the LAD, onset to door time more than 3 hours and not on regular medications were significant adverse predictors of death due to all causes and death due to cardiovascular causes (Table 4). An increase in the ejection fraction by $1 \%$, reduced the cardiovascular mortality $(\mathrm{HR}=0.76,95 \%$ CI 0.66-0.87; $p=0.01$ ).

When deaths due to all causes and deaths due to cardiovascular causes were modelled using the Cox's proportional Hazards model using a stepwise procedure, occlusion of the proximal segment of the LAD was the only significant predictor for both deaths due to all causes $(\mathrm{HR}=10.98 ; p=0.04)$ and for deaths due to cardiovascular causes $(\mathrm{HR}=12.00 ; p=0.03)$.

Table 1. Demographic and clinical characteristics of the study population

\begin{tabular}{|c|c|}
\hline & $N(\%)$ \\
\hline \multicolumn{2}{|l|}{ Age in years, mean $( \pm \mathrm{SD})$} \\
\hline Male & $55.3(9.2)$ \\
\hline Female & $58.8(7.1)$ \\
\hline \multicolumn{2}{|l|}{ Sex } \\
\hline Male & $153(77.7)$ \\
\hline Female & $44(22.3)$ \\
\hline \multicolumn{2}{|l|}{ History of alcohol consumption } \\
\hline Ever or current use & $130(66.0)$ \\
\hline Never used & $67(34.0)$ \\
\hline \multicolumn{2}{|l|}{ History of smoking } \\
\hline Ever or current smoker & $97(49.2)$ \\
\hline Never smoker & $100(50.8)$ \\
\hline \multicolumn{2}{|l|}{ Co-morbidities } \\
\hline Hypertension & $139(70.5)$ \\
\hline Hypercholesterolemia & $147(74.6)$ \\
\hline Diabetes mellitus & $107(54.3)$ \\
\hline \multicolumn{2}{|l|}{$\mathrm{BMI}\left(\mathrm{Kg} / \mathrm{m}^{2}\right)$, mean $( \pm \mathrm{SD})$} \\
\hline Male & $28.5(4.8)$ \\
\hline Female & $27.4(3.2)$ \\
\hline HBA1C $(\%)$, mean $( \pm \mathrm{SD})$ & $6.8(1.6)$ \\
\hline Serum cholesterol level $(\mathrm{mg} / \mathrm{dl})$, mean $( \pm \mathrm{SD})$ & $219.9(38.9)$ \\
\hline LDL (mg/dl), mean $( \pm \mathrm{SD})$ & $140.5(30.4)$ \\
\hline $\mathrm{HDL}(\mathrm{mg} / \mathrm{dl})$, mean $( \pm \mathrm{SD})$ & $55.2(9.1)$ \\
\hline Triglyceride level (mg/dl), mean $( \pm \mathrm{SD})$ & $196.3(39.5)$ \\
\hline Cholesterol / HDL ratio, mean $( \pm \mathrm{SD})$ & $4.2(1.1)$ \\
\hline
\end{tabular}

Table 2. Presenting features and other clinical characteristics type of stent used

\begin{tabular}{lc}
\hline & $N(\%)$ \\
\hline $\begin{array}{l}\text { Onset to door time } \\
<\text { one hour }\end{array}$ & $31(15.7)$ \\
1 hour -2 hours & $34(17.3)$ \\
2 hour -3 hours & $120(60.9)$ \\
$>3$ hours & $12(6.1)$ \\
\hline Door to balloon time & \\
$<1 / 2-$ hour & $165(83.8)$ \\
$1 / 2-1$ hour & $32(16.2)$ \\
\hline Symptoms & $124(62.9)$ \\
Typical symptoms & $73(37.1)$ \\
Atypical symptoms & \\
\hline
\end{tabular}

Vital status at the time of admission

Systolic blood pressure $[\mathrm{mmHg}]$, mean $( \pm \mathrm{SD}) \quad 135.5(21.6)$

Diastolic blood pressure $[\mathrm{mmHg}]$, mean \pm SD) $\quad 89.4(17.4)$

Heart rate [per/min], mean $( \pm \mathrm{SD})$

$91.3(14.7)$

\begin{tabular}{ll}
\hline Ejection fraction & \\
Below $50 \%$ & $62(47.7)$ \\
Above $50 \%$ & $68(52.3)$
\end{tabular}

Killip Class

$\begin{array}{lc}\text { Killip class I } & 124(70.9) \\ \text { Killip class II } & 24(13.7) \\ \text { Killip class III } & 17(9.7) \\ \text { Killip class IV } & 10(5.7)\end{array}$

\begin{tabular}{lc}
\hline Left anterior descending artery $(\mathrm{n}=160)$ & \\
Proximal segment & $47(23.8)$ \\
Middle segment & $77(39.1)$ \\
Distal segment & $36(18.3)$ \\
Left circumflex $(\mathrm{n}=2)$ & $2(1.0)$ \\
Right coronary artery $(\mathrm{n}=35)$ & \\
Proximal segment & $14(7.1)$ \\
Middle segment & $21(10.7)$ \\
\hline
\end{tabular}

\begin{tabular}{lr}
\hline Type of stent & \\
Bare metal stent & $76(38.6)$ \\
Drug eluting stent & $121(61.4)$ \\
\hline
\end{tabular}

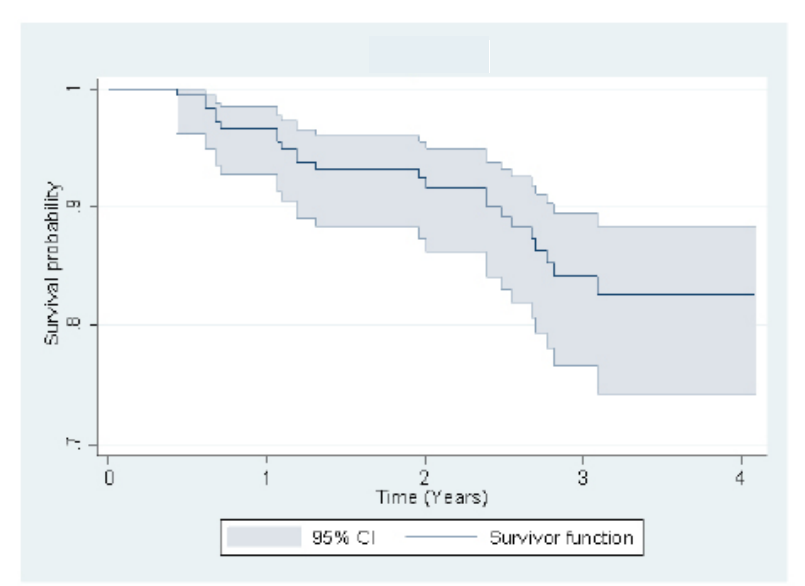

Figure 1. Kaplan-Meier curve for all deaths 
Table 3. Cause of death and site of lesion

\begin{tabular}{lr}
\hline Cause of death & $N(\%)$ \\
\hline All causes & \\
Congestive heart failure & $7(30.4)$ \\
Acute myocardial infarction & $6(26.1)$ \\
Intracranial haemorrhage/stroke & $3(13.0)$ \\
Renal failure & $3(13.0)$ \\
Septicaemia & $2(8.7)$ \\
Others & $2(8.7)$ \\
Cardiac related deaths (n=13) & \\
Congestive heart failure & $7(53.8)$ \\
Acute myocardial infarction & $6(46.2)$ \\
Lesion site in LAD (n=17) & \\
Congestive heart failure & $6(35.4)$ \\
Acute myocardial infarction & $4(23.6)$ \\
Intracranial haemorrhage/stroke & $2(11.7)$ \\
Renal failure & $3(17.6)$ \\
Septicaemia & $2(11.7)$ \\
Lesion site in RCA or Left circumflex $(\mathrm{n}=6)$ & \\
Congestive heart failure & $1(16.6)$ \\
Acute myocardial infarction & $2(33.3)$ \\
Intracranial haemorrhage/stroke & $1(16.6)$ \\
Others & $2(33.3)$ \\
\hline
\end{tabular}

Table 5 shows the multivariate predictors of long term mortality of patients who had a culprit lesion in the right coronary artery or the left circumflex artery. Not being on regular medication (adjusted $\mathrm{HR}=4.39 ; p=0.01$ ) and higher ejection fraction (adjusted $\mathrm{HR}=0.92 ; p=0.03$ ) were significant predictors of mortality due to all causes. Patients not on regular medication (adjusted $\mathrm{HR}=5.39$; $p=0.03$ ), higher ejection fraction (adjusted $\mathrm{HR}=0.80$; $p=0.01$ ) and onset to door time more than 3 hours (HR: $8.35 ; p=0.02)$ were significant predictors of mortality due to cardiovascular causes.

\section{Discussion}

We report in STEMI patients following PTCA 83\% three years survival probability of deaths due to all causes and $89 \%$ probability of surviving deaths due to cardiovascular causes. These are comparable to findings reported from centres of excellence in developed countries.

In our series, there were more males. Similar results have been reported elsewhere. In the Euro Heart Study males were predominant among patients less than 55 years [13]. In the US, males have a higher risk of having STEMI than females [14]. The incidence and presentation

Table 4. Unadjusted predictors of long term mortality of the study population

\begin{tabular}{|c|c|c|c|c|}
\hline Variable & $\begin{array}{l}\text { Death due to all causes } \\
\text { Unadjusted hazard ratio } \\
\qquad(95 \% \text { CI) }\end{array}$ & $p$-value & $\begin{array}{c}\text { Death due to } \\
\text { cardiovascular causes } \\
\text { Unadjusted hazard ratio } \\
(95 \% \mathrm{CI})\end{array}$ & $p$-value \\
\hline Age (years) & $1.05(1.00-1.10)$ & 0.04 & $1.04(0.97-1.10)$ & 0.24 \\
\hline Male sex & $1.25(0.46-3.38)$ & 0.65 & $1.94(0.42-8.78)$ & 0.40 \\
\hline Smoking & $2.27(0.96-5.38)$ & 0.06 & $4.01(1.10-14.59)$ & 0.03 \\
\hline Alcohol consumption & $1.37(0.56-3.34)$ & 0.48 & $1.98(0.54-7.20)$ & 0.29 \\
\hline Diabetes & $2.32(0.91-5.89)$ & 0.07 & $4.41(0.97-19.91)$ & 0.04 \\
\hline Dyslipidaemia & $4.39(1.03-18.74)$ & 0.04 & $9.65(1.25-74.28)$ & 0.02 \\
\hline Hypertension & $1.41(0.99-18.02)$ & 0.50 & $2.17(0.48-9.82)$ & 0.31 \\
\hline \multicolumn{5}{|l|}{ Onset to door time (hours) } \\
\hline More than $1 \mathrm{~h}<2 \mathrm{~h}$ & $1.05(0.15-7.46)$ & 0.96 & $1.08(0.07-17.27)$ & 0.95 \\
\hline More than $2 \mathrm{~h}<3 \mathrm{~h}$ & $2.05(0.46-9.04)$ & 0.34 & $2.43(0.31-19.52)$ & 0.41 \\
\hline More than $3 \mathrm{~h}$ & $8.59(1.66-44.42)$ & 0.01 & $10.94(1.33-105.81)$ & 0.03 \\
\hline Positive - HFABP & $5.30(1.17-23.96)$ & 0.03 & $5.30(1.17-23.95)$ & 0.03 \\
\hline Positive - TropI & $1.07(0.35-3.26)$ & 0.91 & $1.08(0.34-3.28)$ & 0.90 \\
\hline Door to balloon time more than $1 / 2$ hour & $1.63(0.64-4.14)$ & 0.30 & $3.94(1.32-11.75)$ & 0.01 \\
\hline Lesion-proximal segment of LAD & $2.45(1.05-7.19)$ & 0.03 & $8.28(1.59-43.07)$ & 0.01 \\
\hline Lesion-proximal segment of RCA & $9.31(1.01-82.56)$ & 0.05 & $9.31(1.01-82.56)$ & 0.05 \\
\hline No regular medication & $5.97(2.94-16.54)$ & 0.01 & $11.94(3.93-35.91)$ & 0.02 \\
\hline Ejection fraction & $0.89(0.83-0.96)$ & 0.01 & $0.76(0.66-0.87)$ & 0.01 \\
\hline \multicolumn{5}{|l|}{ Killip class classification* } \\
\hline Killip class II & $0.88(0.20-3.91)$ & 0.87 & $1.11(0.21-8.83)$ & 0.95 \\
\hline Killip class III & $1.78(0.51-6.28)$ & 0.36 & $4.11(0.93-16.84)$ & 0.06 \\
\hline Killip class IV & $2.78(0.79-9.68)$ & 0.11 & $4.21(0.85-20.90)$ & 0.08 \\
\hline Post MI associated with complications & $6.65(2.80-15.79)$ & 0.01 & $14.13(4.46-43.22)$ & 0.001 \\
\hline
\end{tabular}

*Reference category Killip class I 
Table 5. Multivariate predictors of long term mortality of patients who had a culprit lesion in the right coronary artery or the left circumflex artery

\begin{tabular}{lcccc}
\hline & $\begin{array}{c}\text { Death due to all causes } \\
\text { Unadjusted hazard ratio } \\
(95 \% \text { CI })\end{array}$ & $\begin{array}{c}\text { p-value } \\
\text { Ejection fraction }\end{array}$ & $\begin{array}{c}\text { Death due to } \\
\text { cardiovascular causes } \\
\text { Unadjusted hazard ratio } \\
(95 \% \text { CI) }\end{array}$ & $\begin{array}{c}p \text {-value } \\
(0.85-0.91)\end{array}$ \\
Not on regular medication & 4.39 & 0.03 & 0.80 & $(0.71-0.91)$ \\
& $(1.65-11.67)$ & 0.01 & 5.93 & 0.01 \\
Onset to door time more than 3 hours & - & - & $(1.20-29.21)$ & 0.03 \\
& & $(1.40-49.84)$ & 0.02 \\
\hline
\end{tabular}

of cardiovascular diseases differ between men and women possibly due to the protective effect of oestrogen. In addition, the skewed distribution of risk factors such as smoking and alcohol consumption among males in Sri Lanka may have resulted in higher male preponderance among STEMI patients. More than $50 \%$ of our study population had known risk factors.

Of our patients $37 \%$ presented with atypical angina type chest pain. Population-based studies and studies based on hospital registries in the USA have reported that the incidence of angina type symptoms is greater among women [15]. The difference in presentation may relate to differences in thrombotic and fibrinolytic activity or differences in the extent and severity of coronary disease and the presence of collateral blood flow [16]. The male preponderance in our study may explain the large percentage of patients presenting with atypical angina type chest pain. In our study $82 \%$ of STEMI patients had left anterior descending artery occlusion. In a series from Iran, similar results were reported where the left anterior descending (LAD) artery was the culprit artery in $84 \%$ of patients [17].

There are many factors that affect long term outcome of STEMI patients who undergo PTCA [13]. We found that age, diabetes, dyslipidaemia, onset of symptoms to door time, history of smoking, positive HFABP, site of LAD occlusion, poor clinical follow-up and low ejection faction were significant predictors of poor long term outcome of STEMI patients undergoing PTCA. In the multivariate analysis, the only significant predictor of 3 -year survival was occlusion of the proximal segment of LAD. After adjusting for the occlusion site of the LAD as a risk factor, low ejection fraction of the heart, patients who were not on regular medication and onset to door time more than three hours were independent risk factors which predicted higher mortality during the three year period of observation of this population.

In our study, door to balloon time was not associated with long term survival of patients treated with PTCA following STEMI contrary to other studies done elsewhere. In one study, a door to balloon time of less than 90 minutes had shown better long term outcome of patients [18]. We may not have seen this effect as all our patients underwent PTCA within 90 minutes of admission. Furthermore according to this study there was a high risk of longer term mortality in Killip class II, II and IV compared to I. But it was not statistically significant. Studies show that therapeutic development and timely intervention of primary coronary interventions (PCI) had significantly reduced the longer term mortality among the STEMI patients even though they were in the higher Killip classes [19, 20].

Mortality rates from NCDs are currently $20-50 \%$ higher in Sri Lanka than in developed countries. The mean age of our sample of STEMI patients is less than 60 years and therefore is necessary to strengthen secondary preventive strategies to prevent premature deaths in the short and the medium-term.

\section{Limitations of the study}

The sample includes patients admitted to a fee levying hospital, hence they may not represent the average Sri Lankan population. Furthermore ten patients had to be excluded from the analysis as they could not proceed with further care at the institute due to financial difficulties.

\section{Conclusions}

There was a male preponderance among STEMI patients. The 3 -year survival probability was $83 \%$ for deaths due to all causes and $89 \%$ for deaths due to cardiovascular causes, LAD occlusion was seen in $80 \%$ of patients with STEMI. Proximal segment LAD occlusion was the only significant predictor of poor prognosis (for both deaths due to all causes and deaths due to cardiovascular causes) after adjusting for other variables. Low ejection faction, symptoms to door time more than three hours and irregular follow up were predictors of mortality in patients who had culprit lesions in the RCA and left circumflex artery. 


\section{Acknowledgements}

We acknowledge the assistance given by the staff of the Nawaloka Hospital PLC, Colombo, Sri Lanka.

\section{References}

1. Mendis S, Puska P, Norrving B. Death and disability due to CVDs. Global Atlas on cardiovascular disease prevention and control. Geneva: WHO, 2011: 8-14.

2. Suraj AA. Diagnosis of Acute Coronary Syndrome. University of California, San Diego, School of Medicine, La Jolla, California. Journal of American Academy of Family Physician 2005; 72: 119-26.

3. Lloyd JD, Adams R, Carnethon M, et al. Heart disease and stroke statistics update: a report from the American Heart Association Statistics Committee and Stroke Statistics Subcommittee. Circulation 2009; 123: 119-21.

4. Ravi PRE, Chamara A, Lara B, et al. NCD burden and trends. An Assessment of the Burden, Issues and Policy Options in Curative Care Services Delivery and Non-communicable Diseases in Sri Lanka. Institute for health policy of Sri Lanka 2010: 18-69.

5. Scott MG, Diane B, Luther TC, et al. Detection, Evaluation, and Treatment of High Blood Cholesterol in Adults. Executive Summary of the third report of The National Cholesterol Education Program (NCEP). USA. Journal of the American Medical Association 2001; 285: 2486-97.

6. Bahendeka S, Colagiuri S, Mendis S. Diagnosis and management of type 2 diabetes in primary health care in low-resource settings. Prevention and Control of Noncommunicable Diseases: Guidelines for primary health care in low-resource settings. Geneva: WHO, 2012: 9-35.

7. World Health Organization and International Society of Hypertension Writing Group. 2003 World Health Organization (WHO)/International Society of Hypertension (ISH) statement on management of hypertension. Geneva. Journal of Hypertension 2003; 21: 1983-92.

8. Parakh K, Thombs BD, Bhat U, et al. Long-term significance of Killip class and left ventricular systolic dysfunction. American Journal of Medicine 2008; 121: 1015-8.

9. Sejersten M. The relative accuracies of ECG precordial lead waveforms derived from EASI leads and those acquired from paramedic applied standard leads. Journal of ElectroCardiology 2003; 36: 179-85.
10. Drew BJ, Califf RM, Funk M, et al. Practice standards for electrocardiographic monitoring in hospital settings. Journal of Cardiovascular Nursing 2005; 20: 76-106.

11. Drew BJ, Califf RM, Funk M, et al. AHA Scientific Statement: Practice standards for electrocardiographic monitoring in hospital settings. Circulation 2004; 110: 2721-46.

12. Smith SW. ST elevation in anterior acute myocardial infarction differs with different methods of measurement. Academic Emergency Medicine 2006; 13: 406-12.

13. Annika R, Lars W, Maarten S, et al. Age, clinical presentation, and outcome of acute coronary syndromes in the Euro heart acute coronary syndrome survey. European Heart Journal 2006; 27: 789-95.

14. Véronique LR. Trends in Incidence, Severity and Outcome of Hospitalized Myocardial Infarction. USA. Circulation 2010; 121: 863-9.

15. Cunningham MA, Lee TH, Cook EF, et al. The effect of gender on the probability of myocardial infarction among emergency department patients with acute chest pain: USA. Journal of General Internal Medicine 1989; 4: 392-8.

16. Hiroki S, Yoshihisa N, Takeshi M, et al. Association of onset to balloon and door to balloon time with long term clinical outcome in patients with ST elevation acute myocardial infarction having primary percutaneous coronary intervention: observational study Japan. BMJ 2012; 344: $1-14$.

17. Heitzler VN, Babić Z, Milicić D, et al. Evaluation of importance of door-to-balloon time and total ischemic time in acute myocardial infarction with ST-elevation treated with primary percutaneous coronary intervention. Croatia. Journal of Acta Clinica Croatica 2012; 51: 387-95.

18. Tsai JP, Chen YC, Hung CL, et al. Reduced In hospital Mortality in Patients with Acute Myocardial Infarction after Practice of Percutaneous Coronary Intervention at a Remote Hospital in Taiwan. Journal of Acta Cardiologica Sinica 2011; 27: 86-93.

19. Takamichi M, Tohru O, Eijirou H, et al. Hospital Physical Activity Status Affects In-hospital Course of Elderly Patients with Acute Myocardial Infarction. Japan. Journal of Medical and Dental Sciences 2010; 57: 119-26.

20. Michael E, Kyoko O, Navaratne KV, et al. Evolving Disease Burden and Risk Factors. Prevention and Control of selected Chronic NCDs in Sri Lanka. Policy Options and Action. The World Bank, 2010: 7-19. 